
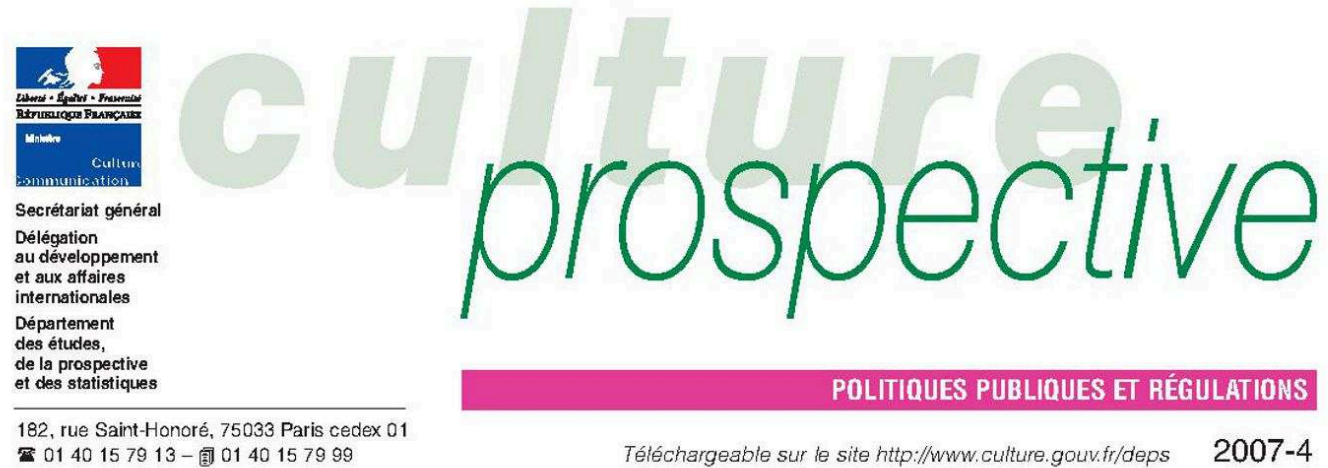

POLITIOUES PUBLIQUES ET RÉGULATIONS

\title{
Régulations du travail artistique
}

Francine Labadie et François Rouet ${ }^{1}$

\author{
Sommaire \\ Le travail artistique face à l'économie de la création ... 3 \\ L'impact sur les acteurs des filières culturelles ........................ 4 \\ Domination croissante de l'aval ........................................ 4 \\ Convergence des filieres \\ et domination accrue des producteurs ……......................... 5 \\ L'hybridation des méthodes de valorisation ........................... 6 \\ Diversification \\ des modes de valorisation et de rémunération ....................... \\ La gestion de l'aléa ............................................................. 7 \\ Valeurs et risques : partage et déports ................................... 8
}

Un cadre juridique

du « travail artistique » en tension

Une adaptation juridique délicate

pour des raisons structurelles?

Hétérogénéité des catégories juridiques

Le travail de création artistique à la croisée de deux droits $\quad 9$

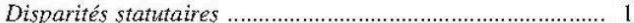

La continuité des droits sociaux comme perspective? ......... 11

Une adaptation juridique nécessaire

pour réguler les relations contractuelles

... dans le cadre de la propriété intellectuelle ....................... 13

Répartition des risques et rémunérations ........................... 14

À l'avenir, quel rôle

pour les mécanismes collectifs et pour l'État? ..................... 17

Un système de relations professionnelles

aux dynamiques peu structurantes

Des démarches collectives

qui se cherchent chez, les non-salariés ............................... 18

Liaction collective

via la gestion collective des droits d'auteur

L'État, arbitre des régulations du travail artistique?

\section{Regulating work in the arts}

\section{Avant-propos}

La synthèse prospective présentée dans ce numéro résulte des travaux du groupe de prospective «Orféo », commencés en 2004 au sein du Commissariat général du Plan, devenu Centre d'analyse stratégique, et mis en débat dans un séminaire interne au Ministère de la culture fin 2006-mi-2007. Ces travaux sont le fruit d'une réflexion collective des professionnels et des experts du secteur.

Cette synthese aborde la grande complexité des modes de régulation du travail de création, et ainsi leur cohérence, alors que la notion de «travail artistique » reste en débat. Partant de l'hypothèse qu'une approche transversale aux secteurs (spectacle vivant et audiovisuel, musique, arts plastiques...) est de nature à ouvrir suffisamment le champ de réflexion, elle impose d'analyser les questions essentielles: rémunération, protection, caractère durable de la création, conditions de développement de la production..., par-delà les différences évidentes de situations, de métiers, de contextes économiques, techniques et juridiques.

Cette hypothèse fait aussi le pari d'une synthèse inachevée qui favorise un débat d'expertise requérant une approche pluridisciplinaire - économie, sociologie, droits sociaux et droit de la propriété littéraire et artistique - et quicherche à poser quelques jalons nouveaux pour continuer d'accompagner la réflexion sur les mutations de la création artistique et de son économie ${ }^{2}$.

P. C.

1. Respectivement chargée de mission au Defem et chargé d'études au DePs (Ministère de la culture, DDA]).

2. Les thèmes présentés dans cette livraison de «Culture prospective» ont été approfondis lors des $2^{e s}$ Journées d'économie de la culture, organisées par le Deps au centre Georges Pompidou les 11 et 12 octobre 2007, "Travail artistique et économie de la creation : protection, valorisation, régulation ». 


\section{Régulations du travail artistique}

Regulating work in the arts

Francine Labadie et François Rouet

Éditeur : Département des études, de la prospective et des statistiques

Lieu d'édition : Paris

Année d'édition : 2007

Date de mise en ligne : 21 septembre 2015

Collection : Culture prospective

ISBN électronique : 9782111398061

\section{Sobooks}

http://books.openedition.org

\section{Édition imprimée}

Date de publication : 1 octobre 2007

Nombre de pages : 20

\section{Référence électronique}

LABADIE, Francine ; ROUET, François. Régulations du travail artistique. Nouvelle édition [en ligne]. Paris : Département des études, de la prospective et des statistiques, 2007 (généré le 25 avril 2021).

Disponible sur Internet : <http://books.openedition.org/deps/869>. ISBN : 9782111398061.

Ce document a été généré automatiquement le 25 avril 2021.

(C) Département des études, de la prospective et des statistiques, 2007

Creative Commons - Attribution - Pas d'Utilisation Commerciale 3.0 non transposé - CC BY-NC 3.0 
L'économie de la création repose sur le travail artistique et le potentiel de renouvellement créatif des auteurs, artistes, créateurs. Or les conditions dans lesquelles les filières culturelles valorisent le travail artistique se trouvent remises en cause par les mutations sociales, économiques et techniques. Au-delà de la reconfiguration des filières, ces évolutions viennent en particulier modifier les conditions dans lesquelles se rémunèrent les acteurs et s'articulent leurs prises de risques respectives. Cette nouvelle donne interroge le cadre juridique du travail de création constitué principalement par le droit social et le droit de la propriété littéraire et artistique.

Le droit social, et particulièrement le droit du travail, ne semblent plus en capacité de jouer leur fonction par essence protectrice : l'équilibre recherché entre liberté du travail d'une part et sécurité d'autre part est ébranlé par la facilitation du recours au contrat à durée déterminée d'usage dans le droit positif ; par ailleurs l'instrumentalisation de l'assurance chômage ne règle pas de manière satisfaisante la couverture des périodes intertravail ; les disparités en termes de droits sociaux entre artistes salariés et artistes non salariés, pourtant en situation de dépendance économique, apparaissent de plus en plus injustifiées au regard des nouvelles conditions d'exercice professionnel ; la continuité des droits sociaux dans un système où se développe la pluriactivité n'est pas assurée.

Du côté du droit de la propriété littéraire et artistique, la contractualisation dans un rapport de forces inégal entre les parties amène à s'interroger sur la manière dont les conditions de cession de droits patrimoniaux sont encadrées en ce qui concerne leur nature, leur étendue et leur durée ainsi que sur la rémunération d'exploitations secondaires de plus en plus nombreuses. Signe des limites de cet encadrement, les pratiques de forfaitisation de la rémunération se développent de manière importante, en particulier dans le cadre d'accords collectifs.

Il s'ensuit des besoins renouvelés de régulation auxquels l'action collective comme l'action publique pourraient à l'avenir davantage contribuer. Il s'agit en effet de répondre de manière adéquate d'une part aux besoins de sécurisation des personnes tout au long de leurs parcours professionnels et d'autre part aux attentes d'une juste rémunération de leur contribution à la production artistique qui tienne compte de l'activité de création dans sa globalité (travail et œuvre).

The economics of artistic creation depend not only on the work done by artists but also on the artists', authors' and creators' capacity for creative renewal. At present, however, the system by which the different branches of culture attribute value to artistic activity is being challenged by social, economic and technical changes. The changes, in addition to altering the structure of these branches, are modifying artists' terms of remuneration and their outlook for taking risks. The new deal calls the juridical framework - consisting basically in labour legislation and literary and artistic property rights - into question.

Social law, labour legislation more particularly, no longer seems able to play its essentially protective role. The required balance between working freedom and work security has been upset by easier recourse to fixed-term contracts, as provided for by positive law. The use of unemployment insurance as an instrument does not satisfactorily solve the problem of cover for out-of-work periods. Welfare rights disparities between salaried and non-salaried artists, even though the latter are economically dependent, appear increasingly unjustified in view of the modern conditions in which professions are exercised. Continuity of welfare rights in a system of growing multi-activity is not assured.

Where literary and artistic property law is concerned, recourse to contracts where the balance of force among the parties is unequal leads to questioning the way in which the surrender of property rights is legally circumscribed as regards their nature, extent, duration and retribution for the ever more numerous forms of secondary exploitation. One sign of the limits of legal protection is the spreading habit of fixed fee payment, especially in the context of collective agreements. 
It may be concluded that a need exists for new regulation and that collective and government action alike could contribute more to bringing it into being. At issue is a satisfactory response to a person's need for security throughout her or his working career and to the expectation of fair pay, which takes account of creative activity in its entirety (labour and product), for contributing to artistic achievement.

FRANCINE LABADIE

chargée de mission au DEFEM

\section{FRANÇOIS ROUET}

Chargé d'études au DEPS 
SOMMAIRE

\section{Avant-propos}

Philippe Chantepie

Régulations du travail artistique

Le travail artistique face à l'économie de la création

Un cadre juridique du « travail artistique » en tension 


\title{
Avant-propos
}

\author{
Philippe Chantepie
}

1 La synthèse prospective présentée dans ce numéro résulte des travaux du groupe de prospective "Orféo », commencés en 2004 au sein du Commissariat général du Plan, devenu Centre d'analyse stratégique, et mis en débat dans un séminaire interne au Ministère de la culture fin 2006-mi-2007. Ces travaux sont le fruit d'une réflexion collective des professionnels et des experts du secteur.

2 Cette synthèse aborde la grande complexité des modes de régulation du travail de création, et ainsi leur cohérence, alors que la notion de «travail artistique » reste en débat. Partant de l'hypothèse qu'une approche transversale aux secteurs (spectacle vivant et audiovisuel, musique, arts plastiques...) est de nature à ouvrir suffisamment le champ de réflexion, elle impose d'analyser les questions essentielles : rémunération, protection, caractère durable de la création, conditions de développement de la production..., par-delà les différences évidentes de situations, de métiers, de contextes économiques, techniques et juridiques.

3 Cette hypothèse fait aussi le pari d'une synthèse inachevée qui favorise un débat d'expertise requérant une approche pluridisciplinaire-économie, sociologie, droits sociaux et droit de la propriété littéraire et artistique - et qui cherche à poser quelques jalons nouveaux pour continuer d'accompagner la réflexion sur les mutations de la création artistique et de son économie ${ }^{1}$.

\section{NOTES}

1. Les thèmes présentés dans cette livraison de «Culture prospective » ont été approfondis lors des $2^{\text {èmes }}$ Journées d'économie de la culture, organisées par le DEPS au centre Georges Pompidou les 11 et 12 octobre 2007, «Travail artistique et économie de la création : protection, valorisation, régulation ». 


\title{
Régulations du travail artistique
}

\author{
Regulating work in the arts
}

1 Récemment, la question du travail artistique, de sa protection et de sa valorisation, est revenue au premier plan dans le débat public sur la culture et son économie. Ce regain d'intérêt s'inscrit dans un contexte d'essor de l'économie de l'immatériel, dont la créativité constitue le levier. Cette dernière peut pourtant, dans le domaine culturel, devenir un maillon faible, tant la domination des acteurs de l'aval au sein des filières des industries culturelles transforme les modes de valorisation de la création. De plus, les évolutions à l'œuvre dans nombre de secteurs d'activité, concernant l'agencement de la production et la flexibilisation de l'organisation du travail, ont un impact sur le système de protection et font surgir l'enjeu de la "flexicurité ".

2 Or, si l'activité de création était hier appréhendée au travers de son résultat - l'œuvre comme " envers du travail" ", l'hypothèse inverse prévaut aujourd'hui :

« Loin des représentations romantiques, contestataires ou subversives de l'artiste, il

faudrait regarder le créateur comme une figure exemplaire du nouveau travailleur,

figure à travers laquelle se lisent des transformations aussi décisives que la

fragmentation du continent salarial, la poussée des professionnels autonomes,

l'amplitude et les ressorts des inégalités contemporaines, la mesure ou l'évaluation

des compétences ou encore l'individualisation des relations d'emploi ${ }^{3}$.»

Ainsi, la notion de «travail de création » - ou travail artistique - s'est développée dans les analyses scientifiques. Elle désigne à la fois le processus complexe d'activité artistique qui mobilise une diversité d'intervenants, suscitant une multitude de démarches et de pratiques, et l'œuvre comme résultat de ce travail. Aujourd'hui, « les deux paradigmes de l'artiste - confondu à une œuvre ou confondu à une activité doivent être considérés en permanence ${ }^{4}$ ". S'affirment donc la fonction de producteur à côté de celle de créateur, l'activité à côté de l'œuvre 5 .

4 L'état des droits, que le droit communautaire est venu depuis peu compléter, est le fruit d'histoires parallèles de droits économiques et sociaux établis pour des filières spécifiques, en lien avec des modèles économiques particuliers, pour des professions/ des individus marqués par des logiques corporatistes et/ou syndicales souvent fortes. Deux principales branches juridiques coexistent - le droit de la propriété littéraire et artistique (PLA) et le droit du travail -, qui ne reconnaissent que de manière très limitée les liens entre œuvre et activité. Ces deux droits renvoient à deux figures 
distinctes de l'artiste : d'un côté, la figure du créateur-auteur, qui est prise en compte par le droit de la propriété littéraire et artistique justifié par le droit de propriété, la protection de la personnalité, l'incitation à la création et la facilitation des échanges ${ }^{6}$, mais pas par l'objectif de conférer un statut à l'artiste en tant que travailleur ${ }^{7}$;

L'économie de la loi du 11 mars 1957 repose sur une propriété privative permettant de déterminer les conditions d'exploitation des œuvres à travers deux types de prérogatives: le droit moral, dont la finalité est de protéger la personnalité de l'auteur et l'intégrité de son œuvre, les droits patrimoniaux qui permettent à l'auteur d'autoriser ou d'interdire les différents modes d'exploitation contre rémunération en principe proportionnelle. Elle est complétée par la protection légale relative aux droits voisins (loi du 3 juillet 1985) conférée à certains « auxiliaires de la création »: artistes interprètes, producteurs de phonogrammes et de vidéogrammes, entreprises de communication audiovisuelle.

de l'autre, sous la figure de travailleur, l'artiste du spectacle ${ }^{8}$, qui bénéficie en France du statut du salariat (voir encadré p. 3). Ce modèle s'est imposé en France ${ }^{9}$ en raison de la force de ses référentiels fondés sur les droits économiques et sociaux avec une finalité de sécurité. La garantie de ressources procurée par le salariat et la protection sociale associée ont rendu ce modèle attractif pour nombre de travailleurs proches de l'indépendance comme les travailleurs artistiques.

La reconnaissance légale de ce statut est effective depuis 1969 au moyen de la " présomption légale de contrat de travail ${ }^{10}$ " puis de « l'assimilation » dans le droit de la sécurité sociale ${ }^{11}$. Cette dernière technique permet, dans un souci de cohérence du « statut social » de l'artiste, de rattacher les « artistes-auteurs ${ }^{12}$ » au régime général de la sécurité sociale (articles L.311-3-5 et L.382-1) par le biais d'une « fiction de salariat ». Sont ainsi assimilés légalement à des travailleurs salariés des travailleurs indépendants - c'est-à-dire non inscrits dans un rapport de subordination juridique - et, par ailleurs, bénéficiant de revenus autres que le salaire (droits d'auteurs, droits voisins, royalties, honoraires ${ }^{13} \ldots$..).

Eu égard à ces caractéristiques propres à chaque système juridique et dans un contexte socio-économique en mutation, comment le droit évolue-t-il pour s'adapter à la nouvelle réalité du travail de création? Deux enjeux sont au centre des tensions que connaissent les modèles juridiques qui encadrent la condition économique et sociale des créateurs.

Dans un contexte où l'artiste tend à se comporter comme son propre entrepreneur, où ses relations avec son employeur ou « donneur d'ordres » sont sporadiques par nature car fondées sur une activité de projet, le premier enjeu concerne les protections accordées aux artistes en contrepartie de l'asymétrie des relations de travail. Deux questions majeures se posent alors :

- comment s'opère la répartition des responsabilités concernant les risques inhérents à la mobilisation du travail ${ }^{14}$ ?

- comment se concilient la nécessité de l'autonomie (et donc de la responsabilité) comme condition de la créativité mais aussi de l'efficacité économique, et la sécurisation de l'exercice professionnel, notamment l'octroi de garanties de ressources?

Le second enjeu concerne la « juste rémunération » du travail artistique. Les niveaux de revenus des artistes sont en effet dépendants de la discontinuité de l'activité, du volume des subventions, d'arbitrages entre dépenses de marketing et dépenses en 
rémunération... et soumis à des pressions à la baisse sans lien avec le travail effectif. Dans le même temps, les sources de revenus se sont diversifiées, marquant la reconnaissance de nouveaux droits et traduisant l'évolution des techniques de reproduction et de diffusion qui permettent aux artistes de combiner différents modes de rémunération sans pour autant toujours augmenter leur niveau global de revenu. Eux-mêmes apportent un certain nombre de réponses aux risques professionnels: mobilité intersectorielle et diversification des activités, autoproduction, etc.

Pour répondre à ces deux enjeux, il apparaît nécessaire de dresser un diagnostic sur les évolutions du travail de création dans le nouveau contexte économique et technique et puis sur leur appréhension par les modes de régulation issue des droits sociaux et des droits de propriété. Examiner les incidences des mutations en cours dans les filières culturelles, en particulier celles des industries culturelles, sur la valorisation du travail de création permet de mettre en lumière les tensions auxquelles ont à faire face le droit social et le droit d'auteur et, dès lors, de chercher à élaborer quelques perspectives, notamment autour de l'action collective comme instrument de régulation.

\section{Le travail artistique face à l'économie de la création}

Les filières culturelles reposent sur le travail artistique et le potentiel de renouvellement créatif des auteurs, artistes, créateurs, dans lesquels leurs partenaires économiques investissent. Une analyse socio-économique du fonctionnement des filières permet d'appréhender les conditions dans lesquelles le travail artistique se valorise et les défis que rencontre cette valorisation. Les mutations des filières culturelles transforment la place et le rôle des acteurs économiques avec trois évolutions qui se renforcent mutuellement: le poids de l'aval dans les filières culturelles, le poids des interrelations croissantes entre filières, le rôle déterminant de la production. De telles évolutions ne peuvent qu'affecter le travail artistique au travers des mutations que connaissent les modalités de sa valorisation.

\section{L'impact sur les acteurs des filières culturelles}

Dans les filières culturelles comme dans toute l'économie, la pression de l'aval (distribution et diffusion) est le phénomène à la fois le plus exogène et le plus déterminant. La domination accrue des producteurs sur l'amont apparaît, pour partie, comme une réaction leur permettant une meilleure maîtrise des risques de production dans un contexte de convergence entre filières.

\section{Domination croissante de l'aval}

12 La place croissante accordée à l'aval par les filières culturelles en fait le stade prééminent et, en conséquence, le vecteur de leurs évolutions. Cette tendance est accentuée par le caractère crucial de la mise en marché pour des productions culturelles qui doivent se commercialiser en référence aux pratiques standard du reste de l'économie. Or, traditionnellement, c'est à l'amont, et à l'autonomie de la création qui s'y manifeste, que la place prééminente est accordée. Il s'ensuit que la tension amont/aval, de nature structurelle et qui se traduit par des crises chroniques dans les différents secteurs, peut apparaitre à présent en voie de se renforcer, passant d'une 
simple opposition culturelle/commerciale à l'affirmation de légitimités antagonistes artistique et culturelle d'une part, marchande et sociale d'autre part -, pourtant tenues de coexister. On observe clairement ce phénomène dans le secteur audiovisuel où le financement publicitaire et la sanction de l'audience sont déterminants et peuvent s'analyser dans le cadre du modèle économique d'un marché biface ${ }^{15}$.

13 L'apparition dans les filières culturelles d'acteurs externes aux mondes de l'art que sont ces filières - opérateurs de télécommunications, industriels de l'informatique, de l'électronique et du logiciel, grande distribution... - et l'ampleur des phénomènes de concentration verticale et horizontale peuvent alimenter la crainte d'une soumission aux seules contraintes de rentabilisation de court terme et d'un appauvrissement subséquent de la création.

Les filières d'industries culturelles font en effet montre d'une concentration horizontale forte, qui leur confère une structure oligopolistique que l'on retrouve d'une filière à l'autre. Elles connaissent également, au moins partiellement, une intégration verticale : édition-diffusion-distribution pour le livre et la musique, de la production à l'exploitation pour le cinéma. En ce qui concerne l'audiovisuel, la réglementation limite cette forme d'intégration. Quant aux arts plastiques, le caractère «aplati » de la filière traduit une prise en charge des fonctions de production-diffusion-commercialisation par les mêmes acteurs - les galeries -, lesquels affirment de plus en plus un rôle de producteurs. De leur côté, les sociétés de ventes aux enchères tendent à développer également une intégration verticale de leurs activités. On retrouve une situation voisine dans les arts de la scène qui connaissent, eux aussi, une faible individualisation de la fonction de diffusion faisant intervenir peu d'acteurs spécifiques à l'exception des tourneurs, tandis que la commercialisation des spectacles est loin d'être l'apanage des lieux, les producteurs ou tourneurs pouvant assumer également cette fonction.

Ces tendances font naître des inquiétudes qui avivent l'enjeu de l'indépendance. Cette notion d'indépendance, quoique ambiguë, est connotée positivement parce qu'elle exprime une capacité à maintenir à distance les logiques financières de court terme, à respecter les logiques professionnelles, à mener un véritable exercice du métier où les professionnels expriment et impriment leur subjectivité, à développer l'autonomie stratégique des entreprises... Le maintien de l'indépendance et d'un tissu de producteurs-éditeurs mais aussi de diffuseurs indépendants - y compris en partenariat mutuellement favorable avec les structures non indépendantes - est perçu comme le garant du renouvellement des divers modes d'expression et du travail artistique luimême.

16 Cette situation contribue en effet à la vitalité et à la pérennité de la frange de l'oligopole dans les filières d'industries culturelles en maintenant une exigence de professionnalisme dans des conditions d'exercice risqué. Elle favorise la diversité dans la nature et les modes de production, et les producteurs indépendants deviennent des interlocuteurs privilégiés pour les travailleurs artistiques débutants. De plus, en contribuant à la diffusion et à l'essaimage de l'innovation dans l'ensemble de la filière pour autant qu'il ne se trouve pas relégué dans une sous-filière indépendante et marginalisée du small is beautiful -, l'exercice indépendant peut constituer un élément clef d'une "écologie de l'innovation». Se développent ainsi des relations et des pratiques complexes de délégation et de sous-traitance dans la définition comme dans la mise en œuvre des projets constitutifs du «cœur de métier» de la production culturelle. 


\section{Convergence des filières et domination accrue des producteurs} de formes extrêmes, comme la quasi-dépendance du cinéma à l'égard de l'audiovisuel compte tenu du rôle essentiel du second dans le financement du premier, à des formes structurelles mais évolutives, comme pour le disque avec la radiodiffusion et le spectacle vivant. Certaines filières permettent à d'autres des valorisations et des exploitations secondaires : le livre pour le cinéma et l'audiovisuel, le cinéma pour le disque par exemple. Ces interrelations s'avèrent cruciales en termes de rentabilisation des succès et parfois de simple rentabilité de la production initiale, comme c'est le cas dans le livre. Leur impact sur le travail artistique est patent car le fait de mobiliser pour partie les mêmes travailleurs artistiques, lesquels relèvent d'une pluralité d'accords collectifs ou de règles juridiques spécifiques, ne facilite pas la gestion de leurs droits et interroge l'identité des métiers. Se profile alors la figure de l'artiste « multi-filières ».

'ensemble des filières (hors arts plastiques) possède en amont, au niveau de la production, un épicentre artistico-professionnel qui mobilise le travail artistique, prend et garde l'initiative en matière de conception des produits et contribue pour partie à créer la demande, quand bien même les producteurs sont en relation avec des donneurs d'ordre en aval, comme les programmateurs. Dans l'économie de projet qu'est l'économie de la création, le jeu offre/demande est particulier : pour certaines productions, il n'y a pas de demande préalable et c'est bien l'offre qui, par ses propositions innovantes, crée la demande. formulent des commandes aux artistes, réalisent des projets qui nécessitent la collaboration de plusieurs d'entre eux, recherchent en permanence des idées éditoriales pour diversifier, enrichir et renouveler leur production et leur offre. Ce recours est important même si une proportion non négligeable de projets n'est finalement pas réalisée pour des raisons d'ordre technique, de débouchés, etc. L'économie de la création est en effet une économie d'abondance des projets ${ }^{16}$.

Dans le même temps, la fonction production/édition s'est hybridée en raison de son rapprochement avec d'autres fonctions constitutives des filières : création/production dans le secteur musical où l'autoproduction est forte ; production/postproduction dans les secteurs du cinéma et de l'audiovisuel ; production/diffusion dans les arts de la scène ; édition/distribution dans les filières de l'écrit. Cette évolution n'est pas sans créer un certain flou sur les contours et les conditions d'exercice des métiers correspondants: responsable littéraire, producteur artistique, producteur délégué, chargé de production... Elle est aussi de nature à renforcer l'asymétrie structurelle entre artistes et producteurs. En effet, même dans le cadre d'une œuvre - c'est-àdire le fruit d'un travail engagé spontanément - où l'artiste se trouve à l'origine de la perspective d'échange avec le producteur, le rapport de force ne lui est favorable que s'il dispose d'une notoriété, d'une spécificité ou d'un succès suffisant qui font qu'on se situe alors dans l'économie de la notoriété et des stars ${ }^{17}$. Pour les autres, qui ont engagé des frais et qui montrent avec force leur attente d'une rentrée financière et de la capacité à en vivre, la négociation est plus difficile.

Dans ce contexte, on observe la montée d'intermédiaires entre créateurs et producteurs, comme celle des managers dans les musiques actuelles ou des agents 
littéraires dans l'édition de livres. La fonction qu'ils s'attribuent est la défense des intérêts des artistes vis-à-vis de la production et leur promotion sur le marché de la notoriété. Ils trouvent d'autant plus d'espace et de légitimité que la relation créateur/ producteur connait des carences et des dysfonctionnements. La gestion collective des droits d'auteur et des droits voisins, en servant d'appui à des démarches et représentations plus collectives des intérêts, permet également de dépasser l'asymétrie à laquelle est souvent confrontée cette relation.

L'évolution des arrangements contractuels entre créateurs et producteurs porte la trace de l'évolution de leurs relations. S'agissant de la valorisation des œuvres, ces arrangements relèvent du droit d'auteur, cadre juridique au sein duquel sont formulées les spécifications des contrats. Cette situation s'applique théoriquement aussi à toutes les formes de commande. Pour les collaborations souvent multiples autour d'un projet, les contrats relèvent de plus en plus à la fois du droit du travail et du droit de la PLA, en termes de droits voisins.

\section{L'hybridation des méthodes de valorisation}

23 Face à la pression de l'aval, l'hybridation des modes de valorisation peut s'interpréter comme un moyen pour l'amont de multiplier et d'articuler ces valorisations dans la perspective d'une efficace gestion de l'aléa et, le cas échéant, de réduire les dépendances vis-à-vis des acteurs de l'aval.

\section{Diversification des modes de valorisation et de rémunération}

Quelles que soient les filières culturelles, les modèles économiques connaissent de profonds bouleversements :

le modèle du marché de l'art, fondé sur la valorisation de l'objet unique, repose sur la vente d'une œuvre et non d'un travail, œuvre pour laquelle il s'agit de construire une demande de rareté et dont la valeur n'est pas directement liée à la quantité de travail nécessaire à son élaboration, ce qui se traduit donc par une double déconnexion entre travail et rémunération. Ce modèle est complété par la participation fréquente des artistes à des activités de formation : à ce titre, leur rémunération est donc issue du salariat ; à cette rémunération s'ajoutent des revenus issus du droit de suite lors des reventes successives des œuvres, sinon du droit d'exposition ;

- le modèle de l'édition, présent largement dans les industries culturelles, repose sur la cession d'un droit sur le fruit du travail artistique et non pas sur le travail en tant que tel, la rémunération étant en principe proportionnelle aux ventes et non à la quantité de travail qui a été nécessaire ;

- le modèle du spectacle vivant, dont le principe est la vente d'un travail ou d'une présence en échange d'une rémunération : relativement moins rentable au regard de la prise de risque assumée dans une économie de marché, il peut avoir besoin d'un soutien publicsubventions - ou privé - mécénat - aux projets ou aux structures et bénéficie de la limitation du coût du travail par l'intermédiaire de la prise en charge par la solidarité interprofessionnelle de la plus grande partie du financement du régime d'indemnisation du chômage ;

- le modèle du spectacle audiovisuel articule des éléments des précédents : la rémunération d'un travail sur le modèle du spectacle vivant et la rémunération d'un droit sur le modèle éditorial. Chacun de ces modèles connaît aujourd'hui des évolutions rapides qui remettent 
en cause les modalités préexistantes de rémunération de la création et, en particulier, complexifient les remontées des droits vers les auteurs ${ }^{18}$;

- le modèle du marché de l'art est confronté au développement d'œuvres, de processus ou d'installations qui ne possèdent plus les attributs d'un objet unique et qui peuvent être valorisés sur un marché de collection. La question de nouvelles formes de rémunération de la création artistique se pose : rémunération d'une performance (travail et présence sur le modèle du spectacle vivant) ou vente d'un droit d'exposition et de représentation des œuvres (modèle éditorial). En outre, ce modèle est touché par le passage progressif à une économie de production comme dans l'économie du cinéma, où l'œuvre n'existe que si des producteurs sont prêts à investir sur un projet, évolution qui élève les niveaux de risque. Se posent également des problèmes contractuels spécifiques pour la répartition des produits de vente lorsque se mêlent financements publics et privés ;

- le modèle du spectacle vivant est, lui, surtout confronté au plafonnement des financements publics et aux limites d'un travail à moindre coût grâce aux systèmes spécifiques d'indemnisation du chômage. Ces financements directs et indirects viennent pérenniser une économie fondée sur les déficits ex ante et finalement une limitation de la rémunération du travail artistique dont rend compte la réduction des « marges artistiques ";

- le modèle éditorial, enfin, est confronté à des modalités de mise à disposition largement renouvelées du son, du texte et de l'image au travers de leur numérisation généralisée et du développement des techniques numériques. Comme il est de plus en plus difficile de rémunérer la création sous forme de droits spécifiques à chaque utilisation ou étape du versioning, le recours à l'idée de vendre non plus un contenu (musique, film) mais un contenant (pochette) sur le modèle du marché de l'art (série limitée, numérotée) ou de vendre des internautes à des annonceurs sur le modèle des médias commerciaux est croissant.

Seul le modèle des médias serait peu affecté parce qu'il repose précisément sur une dynamique entre les deux faces de son marché: le public auquel s'adressent les programmes et les annonceurs à qui est vendue l'audience.

Les différents modèles évoluant simultanément, il est possible qu'on assiste en réalité à un double mouvement :

une bipolarisation autour, d'une part, d'un modèle unique rapprochant celui des arts plastiques et celui du spectacle vivant dans une logique d'« événementialisation », et autour, d'autre part, d'un modèle centré sur celui du spectacle audiovisuel correspondant au basculement plus général de l'économie des contenus dans l'économie de la communication;

l'articulation croissante de ces deux polarités - événement/star-system/notoriété d'une part et, d'autre part, médiatisation des contenus éditoriaux, événementiels et nouveautés par le spectacle audiovisuel et des réseaux numériques -, d'abord dans la logique classique de débouchés du premier pôle vers le second, et d'accès du premier aux ressources du second, et surtout, dans une dynamique plus nouvelle de marchés bifaces assis sur des plates-formes médiatiques, par lesquels l'événement fait l'audience et réciproquement, dans un contexte d'économie d'abondance numérique et de forte concurrence sur la captation des ressources d'attention ${ }^{19}$. 


\section{La gestion de l'aléa}

30 Ce contexte, caractérisé par un jeu non stabilisé de mutations profondes des modèles économiques de la culture, ébranle l'ensemble du système traditionnel de cette économie. En effet, le cœur du système qui tient à la fluidité entre l'amont - où se joue la maîtrise économique et financière du risque artistique et/ou de l'aléa de production - et l'aval, constitué par les différents modes de valorisation, est rendu incertain par le mouvement d'hybridation et de bipolarisation des modèles économiques traditionnels, comme par l'articulation croissante des structures de valorisation des œuvres/produits issus du travail artistique.

31 Traditionnellement, la gestion de l'aléa de production est au cœur de chacune des filières de l'économie de la création. Les techniques de nature à en contrôler les effets sont éprouvées et utilisées de manière renouvelée, à l'instar du star-system ou des blockbusters. En effet, le phénomène des stars prend une importance croissante en vue d'atteindre le succès et de concrétiser les espérances de vente, d'audience et de notoriété, liés souvent à des logiques de superproductions, parfois dans une logique de suites (sequels). C'est notamment l'effet de nouveaux canaux de distribution et de diffusion permis par le numérique. Dans ce contexte, on observe la généralisation de l'inflation des budgets de production, des coûts artistiques (quelques artistes concentrant les cachets), mais surtout des dépenses relatives à la promotion, publicité et marketing.

32 Classiquement encore, la recherche de «nouveauté » est essentielle dans la gestion de l'aléa de production. Pour autant, les marchés demeurent caractérisés par une intrication étroite entre innovation et permanence du côté de l'offre, renvoyant du côté de la demande à l'intrication entre découverte et sécurité. Dans les filières se développent ainsi simultanément des stratégies, respectivement push et pull, de propositions risquées et de réponses aux grandes attentes de la demande. Pour réduire l'aléa de production inhérent à la mise en marché de contenus et tirer parti de la production du "nouveau», différentes stratégies sont à l'œuvre: d'abord, une production numériquement importante apparaissant parfois comme une surproduction, mais aussi une production qui doit trouver son public, moins prévendue, apanage ou niche de repli pour des acteurs de moindre taille se revendiquant souvent comme " indépendants ».

Cette dualité de la production se manifeste particulièrement dans les filières d'industries culturelles, sans correspondre pour autant à l'opposition oligopole/frange. Elle trouve des similitudes dans les filières d'arts plastiques et du spectacle autour des clivages plus ou moins prononcés en fonction de l'accès ou non à la notoriété entre, d'un côté, des productions mobilisant des moyens limités et visant initialement des audiences restreintes et, de l'autre, des productions plus coûteuses destinées à une plus large audience voire au grand public.

La gestion parallèle du rapport nouveauté/catalogue est indispensable à la gestion de l'aléa de production : il faut en effet à la fois attester d'une offre de qualité pour ces nouveaux biens d'expérience que sont les nouveautés et rentabiliser les fonds de catalogues, ce second objectif bénéficiant sans doute d'effets favorables du numérique tant pour la conservation que pour l'accès. Pareilles évolutions confortent d'ailleurs le développement des exploitations secondaires qui se traduit par l'importance croissante des droits annexes ou dérivés dans les modes de valorisation des œuvres. 


\section{Valeurs et risques : partage et déports}

leurs différents stades, sont des espaces de compétition en ce qui concerne le partage de la valeur ajoutée et des retombées du succès et quant aux déports des risques à assumer. De manière concomitante, les acteurs prennent des risques qui s'avèrent comme seconds par rapport au risque, primaire ou initial, portant sur la mise au point au stade de la production, voire de l'édition dans les industries culturelles. La question du niveau de risques à chaque stade se pose mais aussi celle de l'interdépendance des risques supportés par les différents acteurs, qui signe la nature de leurs relations. Ce partage ou déport des prises de risque vaut naturellement aussi pour les travailleurs artistiques à travers leurs rémunérations. Du côté de la production, les niveaux de risques dépendent largement de l'importance des coûts fixes et surtout du fait d'être récupérables ou non en cas d'échec, ce qui rend les modes de rémunération déterminants. Ces derniers sont également essentiels pour expliquer ou traduire l'interdépendance des risques et le partage des fruits du succès entre les différents acteurs selon que les rémunérations sont proportionnelles, forfaitaires, font l'objet de minima garantis, etc.

En matière de gestion du risque, les différentes filières culturelles connaissent de notables évolutions :

- l'ampleur du risque global a tendance à croître dans la filière de la musique enregistrée, du côté des majors, mais aussi dans le cinéma ou pour les livres à succès. Le phénomène tient à l'existence de coûts fixes élevés aux différents stades et en particulier de la diffusion en matière de promotion et de publicité, croissant dans une même spirale de renforcement où des coûts fixes plus élevés sont consentis comme des moyens de rendre le succès moins incertain ou plus important ;

- le niveau de risques de chaque acteur peut également évoluer significativement : ainsi, dans le cinéma, la prise de risque éditoriale du producteur tend à reculer face à des démarches systématiques de préfinancement; dans les arts plastiques, on voit apparaitre une prise de risque "producteur » dans le financement de la réalisation d'œuvres coûteuses ; dans la musique, on observe une prise de risque croissante par l'artiste au travers de l'autoproduction;

- les risques respectifs des différents acteurs apparaissent très liés dans une filière comme celle du livre, où le mode généralisé de rémunération est le pourcentage du prix public fixé par l'éditeur, conduisant les acteurs à accompagner de près la prise de risque éditoriale et ses éventuelles formes de mutualisation;

- l'industrie des jeux vidéo constitue une exception notable par un report du risque sur l'amont - éditeurs, puis studios, puis créateurs -, alors même que la part créative est majeure dans la valeur ajoutée du produit final, et ce, sans reconnaissance de la propriété littéraire et artistique.

Dans un contexte marqué par des mutations aussi profondes, les conditions de mise en œuvre du travail artistique et les arrangements contractuels qui déterminent rémunérations et prise de risque deviennent toujours plus cruciaux. Ceci amène à se tourner vers les cadres juridiques qui les gouvernent afin d'en examiner la cohérence et la capacité à encadrer des pratiques en changement. 


\section{Un cadre juridique du « travail artistique » en tension}

Face à des mutations de cette ampleur et au regard des droits - droits de la propriété littéraire et artistique, droits sociaux et règles conventionnelles collectives ou individuelles - mobilisés pour encadrer les conditions économiques et sociales des acteurs des filières culturelles et les stabiliser, ces différents droits ne peuvent qu'être mis en tension. Sans qu'il s'agisse pour autant d'une remise en cause d'un système juridique forgé dans le temps et le plus souvent par filières ou catégories d'acteurs, ces droits sont appelés à s'adapter aux nouvelles réalités socio-économiques de l'économie de la création.

\section{Une adaptation juridique délicate pour des raisons structurelles?}

39 Le travail artistique est encadré juridiquement par une pluralité de sources de droits Code du travail, de la sécurité sociale, de la propriété littéraire et artistique, Code civil, Code de commerce, Code général des impôts... - relevant de logiques et finalités différentes. S'ajoutent à cet ensemble d'autres règles ayant trait à la création, développées souvent selon des logiques catégorielles et professionnelles : droit du spectacle vivant, droit du cinéma par exemple... Enfin, selon le champ d'activité concerné, un ensemble complexe d'instruments de portée inégale s'applique : chartes, usages professionnels, conventions collectives, etc. Il résulte de cet enchevêtrement un « droit de la création » au caractère foisonnant et complexe.

\section{Hétérogénéité des catégories juridiques}

40 La dispersion des normes se traduit par un cloisonnement des catégories juridiques, celles-ci traitant dès lors les activités artistiques de manière partielle: le Code du travail traite des "artistes du spectacle ${ }^{20 "}$, lesquels sont considérés par le Code de la propriété intellectuelle comme " artistes interprètes ${ }^{21}$ ". Quant aux « employeurs » des " artistes du spectacle", ils ne sont pas définis précisément par le Code du travail ${ }^{22}$, alors que le sont les intermédiaires, ce qui crée une «incomplétude de la relation sociale ${ }^{23}$ ".

41 Dans le Code de la propriété intellectuelle, le donneur d'ordre apparaît sous la figure de l'éditeur/producteur, entendu comme celui qui est «à l'initiative » de la divulgation de l'œuvre et comme

42 «cessionnaire» des droits de l'auteur ou de l'interprète. Les auteurs, eux, sont indirectement définis par ce Code à travers « l'œuvre de l'esprit » dont le code établit une liste non exhaustive. Certes, la qualité d'auteur « appartient à celui ou à ceux sous le nom de qui l'œuvre est divulguée », mais « il ne s'agit là que d'une présomption qui souffre la preuve contraire ", tandis que, à des fins de gestion, les organismes sociaux en ont, pour leur part, une conception plus précise et restrictive au travers des listes qu'ils établissent ${ }^{24}$.

43 L'exercice de délimitation des acteurs de la création peut conduire à des logiques d'exclusion. Prenons le cas des artistes interprètes : travailleurs salariés, ils ne sont pas considérés comme des auteurs mais, aux côtés des producteurs de phonogrammes et vidéogrammes, comme des "auxiliaires de la création", même si leur rôle dans la 
création est avéré. Or, dans le même temps, les traducteurs ou encore les concepteurs de lumières d'un monument public se voient reconnus un droit d'auteur ${ }^{25}$.

\section{Le travail de création artistique à la croisée de deux droits}

En réalité, les deux branches juridiques appréhendent le travail de création à deux moments différents : au moment du processus créatif ou de la production artistique d'une part, au moment où l'œuvre est diffusée et fait l'objet d'une exploitation d'autre part. Logique de la création et logique du travail sont disjointes du fait du cloisonnement des règles concernant l'exploitation de l'œuvre ou des interprétations, ou bien le travail de création: ces règles prennent leurs sources dans des textes distincts, obéissent à une économie propre et s'ignorent en grande partie.

Le Code du travail ne traite du travail artistique qu'à travers le livre VII relatif aux dispositions particulières à certaines professions, c'est-à-dire dans le cadre d'un statut spécial, mais sans préciser les caractéristiques de l'activité de création. L'articulation avec la propriété littéraire et artistique n'est opérée qu'en ce qui concerne la rémunération ${ }^{26}$. En outre, la logique collective présente dans la relation de travail ne s'accorde pas toujours avec les prescriptions du Code de la propriété littéraire et artistique, qui place l'auteur/individu au cœur de la protection. Des difficultés peuvent en résulter au plan contractuel, le Code de la propriété littéraire et artistique investissant l'auteur d'un pouvoir individuel que la négociation collective contrarie parfois. En effet, si la négociation collective est bien présente dans ce code, c'est selon des principes et méthodes distincts ne facilitant pas toujours la conciliation avec les règles issues du Code du travail.

Le Code de la propriété littéraire et artistique définit la création comme « la réalisation de la conception de l'auteur » (art L. 111-2), ce qui suppose une distinction entre travail d'un côté et créativité de l'autre. Le travail s'inscrit dans la réalisation-acte de concrétisation et d'extériorisation - alors que la spécificité de la création ressortit de l'acte de conception par lequel la personnalité s'exprime. Les deux actes sont différents et complémentaires - la créativité est singulière, le travail interchangeable - mais à l'intérieur de l'acte de création, ces composantes se combinent et finissent par s'intégrer. Le Code de la propriété littéraire et artistique n'a pas pourtant cette logique : il ignore le travail, sauf en ce qui concerne la commande pour la publicité et la production audiovisuelle, et privilégie l'œuvre et son exploitation, en sorte que la rémunération de l'auteur dépend du succès économique de cette dernière.

\section{Disparités statutaires}

Cette hétérogénéité au plan juridique non seulement ne favorise pas la prise en compte à la fois de la variété et de l'identité du travail de création artistique, mais est également source de disparités statutaires. La variété des statuts professionnels est l'héritage d'une construction sociale. En effet, le modèle, particulièrement emblématique, de "l'artiste-auteur ", fourni par l'histoire, a longtemps été et reste encore - la référence en la matière, mais sous le double effet de l'institutionnalisation de la culture et de son industrialisation, des métiers traditionnellement individuels se sont progressivement érigés en professions. C'est davantage par assimilation que par un processus de construction autonome que les métiers artistiques sont, pour partie, entrés dans l'univers de la normalisation du travail. 
Ainsi certains artistes accomplissent leur prestation de travail en bénéficiant d'un statut de salarié par détermination de la loi. Au titre de l'État-providence, le salariat, qui s'est imposé en France, a permis aux artistes du spectacle de bénéficier de protection de droit commun ${ }^{27}$, mais ce statut ne concerne essentiellement que les artistes et techniciens $\mathrm{du}$ spectacle vivant, de l'audiovisuel et du cinéma. Juridiquement, il est conféré par présomption de contrat de travail (Code du travail) et par assimilation (Code de la sécurité sociale) sans que l'appréciation de la subordination juridique n'ait été précisément réalisée. Par conséquent, bien qu'ils se comportent comme de véritables indépendants vis-à-vis des entreprises du spectacle qui les emploient ou, à tout le moins, conservent une large indépendance dans l'exécution de leur travail - libre expression de son art, possession de tout ou partie du matériel utilisé, latitude d'employer lui-même une ou plusieurs personnes dès lors que l'artiste participe personnellement au spectacle... -, la relation de travail est présumée relever d'un contrat de travail. Il s'agit toutefois d'une présomption simple et non irréfragable: l'artiste peut choisir le statut d'indépendant (au sens de non-salarié). Enfin, les artistes salariés reçoivent une rémunération sous forme de salaire (cachet), assujettie aux cotisations de sécurité sociale et d'assurance chômage, et bénéficient en retour de la protection afférente.

D'autres ne sont pas salariés alors qu'ils se trouvent dans une dépendance économique manifeste à l'égard de leurs donneurs d'ordre : c'est le cas des écrivains vis-à-vis de leur maison d'édition, des interprètes vis-à-vis de leur maison de disques ou encore des plasticiens vis-à-vis des galeristes lorsque ces derniers assument la fonction de producteur de l'œuvre. Pour ces non-salariés, la relation de travail ne relève pas, même par présomption, du contrat de travail mais du louage d'ouvrage ${ }^{28}$, qui prend la forme d'un contrat d'auteur, d'un contrat d'édition, aux termes duquel le créateur cède ses droits patrimoniaux à l'investisseur aux fins d'exploitation de l'œuvre et reçoit en contrepartie des redevances proportionnelles au succès qu'elle rencontrera. Ces professionnels, bien que non-salariés, peuvent cependant, par « rattachement», être assujettis au régime général de la sécurité sociale, avec des cotisations spécifiques. Ils sont en revanche exclus de la protection contre les accidents du travail et les maladies professionnelles, comme de l'assurance chômage ou du droit à la formation professionnelle continue, leurs donneurs d'ordre, qui ne sont pas assimilés à des employeurs, n'étant pas tenus en effet d'acquitter les cotisations afférentes à ces risques et sécurités.

50 La distinction salariat/indépendance se traduit donc par des inégalités dans la couverture des risques sociaux, dont l'atténuation pourrait passer par des novations juridiques visant à favoriser une meilleure conciliation entre autonomie du travail et sécurisation des parcours professionnels.

\section{La continuité des droits sociaux comme perspective?}

51 À ces inégalités en termes de statut, s'ajoute une complexification croissante des situations sociales individuelles, les professionnels étant de plus en plus souvent appelés à enchaîner voire cumuler simultanément des activités de nature différente. C'est le cas des réalisateurs de cinéma ou des metteurs en scène de théâtre, à la fois auteurs - et rémunérés comme tels - et salariés de l'entreprise qui assure la production de l'œuvre. C'est aussi le cas des écrivains et plasticiens, également confrontés à la discontinuité de leur activité créatrice et ne bénéficiant pas des mêmes garanties 
sociales : ils sont souvent amenés à rechercher ces garanties dans une autre activité salariée, exercée dans un autre champ, et connaissent donc une mixité statutaire de fait. Quant aux artistes interprètes, ils peuvent être rémunérés aussi bien comme salariés que comme bénéficiaires de droits voisins du droit d'auteur. Il en est de même pour les photographes de presse au statut incertain entre droit du travail et droit d'auteur ${ }^{29}$. Ces situations mixtes sont souvent laissées à l'appréciation des parties au contrat qui recherchent la qualification juridique la plus favorable pour elles. De ce fait, ces situations sont susceptibles de requalification amiable ou judiciaire. Et lorsqu'il y a affiliations multiples, particulièrement dans le cas des créateurs à forte mobilité internationale, elles occasionnent un surcoût de protection.

Dans le contexte de l'économie de projets qu'est l'économie culturelle, la continuité des droits sociaux pourrait être de nature à gommer des disparités que les évolutions du travail artistique rendent moins justifiables.

\section{Une adaptation juridique nécessaire pour réguler les relations contractuelles}

Que ce soit dans le cadre du droit du travail ou du droit de la propriété littéraire et artistique, le contrat est un mécanisme de régulation majeur des relations entre les créateurs et leurs employeurs ou les investisseurs, en ce qu'il opère un partage des risques, qu'il s'agisse de risques liés à l'emploi ou de risques économiques. La question est de savoir comment évoluent les équilibres en la matière.

\section{... dans le cadre des droits sociaux}

Depuis plus d'une vingtaine d'années, la relation de travail qui lie un artiste du spectacle à un employeur a considérablement changé. Jusqu'au début des années 1980, le régime d'emploi des travailleurs artistiques était gouverné par un objectif de sécurisation ; s'est forgé depuis un compromis implicite entre liberté de travail et sécurisation, notamment à travers l'instauration d'une garantie de ressources pour les travailleurs par le biais des prestations de chômage (voir encadré ci-après).

Cette évolution s'est opérée avec la loi de janvier 1979 qui a créé le contrat à durée déterminée auquel les secteurs du spectacle et de l'audiovisuel recourent fortement, compte tenu de la nature discontinue de l'activité. En 1982, le législateur a consacré la précarité de l'emploi en reconnaissant qu'il s'agit là de secteurs « où il est d'usage constant de ne pas recourir au contrat à durée indéterminée en raison de la nature de l'activité exercée et du caractère par nature temporaire des emplois » (article L 122-1-1 du Code du travail). Des aménagements apparaissant alors nécessaires pour rétablir une certaine sécurité, la loi fut relayée par le droit conventionnel, précisément par l'accord interbranche (dit « accord Michel ») du 12 octobre 1988 qui encadre le recours au contrat dit « d'usage ». Toutefois si, d'un côté, la loi a favorisé la « libération de l'activité » en organisant la mobilité et la disponibilité des travailleurs du spectacle, et partant, leur pluriactivité, ou plutôt la multiplicité de relations de travail avec différents employeurs, de l'autre, elle s'est articulée avec la négociation collective pour instaurer des contreparties à la précarité de l'emploi, notamment à travers le régime de l'intermittence des travailleurs du spectacle. Bien que le salarié ait librement convenu avec l'employeur de conclure un contrat à durée déterminée, il 
est considéré juridiquement que la précarité est plutôt subie que voulue. C'est ce qui justifie que le travailleur artistique, suite à la cessation du contrat de travail à durée déterminée, ait le droit de demander l'obtention de prestations de chômage, comme tout salarié qui perd involontairement son emploi du fait d'un licenciement. En 1983, les partenaires sociaux font passer le seuil d'indemnisation à 507 heures d'activité au cours des 12 derniers mois (au lieu de 1014 heures sur 18 mois précédemment). Le système de report du risque d'emploi est ainsi mis en place, de l'employeur vers le travailleur du spectacle, du travailleur du spectacle vers la solidarité interprofessionnelle via l'Unedic.

Si le CDD d'usage est l'instrument principal de cette tentative de conciliation, il favorise toutefois une désintégration de la relation d'emploi en contrats fragmentés et fait augmenter le risque de sous-emploi pour le salarié. Il permet en outre de s'affranchir de toutes les limites que comporte le CDD de droit commun (recours pour motif de remplacement d'un salarié ou accroissement d'activité, respect d'une période de carence entre deux CDD successifs, versement d'une indemnité de précarité en fin de contrat).

Malgré le grand succès que connaît ce $\mathrm{CDD}^{30}$, le processus n'est peut-être pas arrivé à son terme au regard de la jurisprudence ${ }^{31}$. Depuis 2003, la Cour de cassation qui, traditionnellement recherchait le caractère temporaire ou permanent de l'emploi afin de requalifier, le cas échéant, une succession de CDD d'usage en CDI, considère désormais qu'il ne relève pas de l'office du juge de rechercher si cette opération n'a pas pour objet de pourvoir un emploi permanent de l'entreprise. Le caractère d'usage constant acquiert ainsi une force nouvelle en devenant le seul critère de légalité. Par ailleurs, il suffit désormais aux employeurs de conclure des accords d'entreprise définissant les emplois pour lesquels il est possible de recourir aux CDD d'usage, qu'ils soient ou non de nature temporaire. Pourtant, des juridictions de premier et second degré continuent d'invoquer l'article L. 122-1 du Code du travail ${ }^{32}$, provoquant en tout état de cause une insécurité juridique.

57 En libérant l'activité ${ }^{33}$, le recours au CDD d'usage permet aux employeurs d'obtenir, du fait de la concurrence sur le marché du travail, « un haut degré d'engagement dans une relation de travail éphémère et non routinière, et une disponibilité de la main-d'œuvre au coût le plus bas, compatible avec l'impératif d'ajustement des investissements à la nature de chaque projet ${ }^{34 "}$.

Cependant, pour les créateurs dans le domaine du spectacle, le risque d'emploi s'est accru malgré le régime d'assurance chômage et le principe de partage équitable du risque fixé dans les "accords Michel ${ }^{35}$ ". Au-delà du recours abusif au CDD d'usage, d'autres stratégies de contournement juridique du Code du travail seraient à l'œuvre. La pratique des contrats fictifs de vente de spectacle semble en effet se développer afin d'éviter de conclure des contrats de travail et, en particulier, de payer les cotisations sociales. Alors que la jurisprudence permettait précédemment de requalifier ces contrats en contrats de travail, la loi du 18 mars 1999, qui définit clairement le rôle des divers entrepreneurs de spectacles, dispose que les diffuseurs n'ont pas la responsabilité d'employeur à l'égard du plateau artistique. 


\section{... dans le cadre de la propriété intellectuelle}

d'ajustement et, pour des motifs analogues, d'évolution du partage des risques est réalisée par la relation contractuelle. La relation entre auteurs et producteurs/éditeurs cherche à atteindre à la fois le juste retour sur investissement éditorial et la mise en perspective d'une carrière. Cette recherche d'équilibre entre intérêts divergents et, par suite, le partage des risques s'effectuent principalement à travers la cession des droits patrimoniaux de l'auteur à l'éditeur/producteur. l'exploitation de l'œuvre, les éditeurs/producteurs sont de facto en position forte dans l'établissement d'un contrat, celui-ci prenant souvent l'aspect d'un contrat "d'adhésion». Seules, les situations où la notoriété individuelle peut assurer une position de négociation plus favorable font exception.

61 La nature de la cession est l'objet de controverses car la loi est très allusive sur ce sujet. S'agitil réellement d'une cession des droits patrimoniaux ou bien d'une cession d'un droit spécifié et déterminé qui autorise l'exploitation, ce que l'on pourrait qualifier en d'autres termes de « licence »? Pour le professeur P. Gaudrat, le droit patrimonial étant un versant de la propriété de l'auteur n'est pas plus cessible que ne l'est le droit moral. Il cite l'article $545 \mathrm{du}$ Code civil : «Nul ne peut être contraint de céder sa propriété, si ce n'est pour cause d'intérêt public et moyennant une juste et préalable indemnité. »

La situation apparaitt plus claire et explicite en droit allemand (loi de 1965) qui procède d'une conception moniste du droit d'auteur : les droits patrimoniaux naissent du droit moral, de la personnalité de l'auteur. Droit moral et droits patrimoniaux forment une unité inséparable, ce qui a pour conséquence que les droits patrimoniaux ne peuvent être cédés, l'auteur ne pouvant accorder que des droits d'utilisation de son œuvre ${ }^{36}$.

Le débat sur la nature de la cession n'est pas que théorique, car nombre d'artistes la vivent en réalité comme une dépossession de propriétée ${ }^{37}$. Leur critique porte d'abord sur le renforcement du déséquilibre dans le partage des risques quand la cession vise tous les droits, y compris les droits dérivés ${ }^{38}$ liés à des exploitations secondaires. Ces droits sont de plus en plus importants compte tenu de la diversification des modes de valorisation et de plus en plus déterminants pour la rentabilité éditoriale. Or, le Code de la propriété littéraire et artistique ne fixe aucune règle de répartition de ces droits entre auteur et éditeur ${ }^{39}$. Rares sont les éditeurs qui, au nom de bonnes pratiques d'adaptation au succès, proposent, au-delà de la publication initiale, un partage plus favorable à l'auteur. Cette situation soulève donc deux problèmes.

63 Avec un rapport de forces inégal, et en dépit des prérogatives de leur droit moral, les auteurs disposent de peu d'armes face à l'éditeur/producteur. Ainsi, actuellement, aucune condition n'est posée à la jouissance des droits de ce dernier, telle que la preuve $d u$ respect du droit des auteurs par exemple. Par ailleurs, rien ne garantit l'auteur du professionnalisme de l'éditeur et ne définit les responsabilités de celui-ci dans le succès ou l'insuccès que rencontrera une œuvre; rien non plus ne précise ses obligations de moyens en matière d'exploitations secondaires. 
Le second problème tient à la portée de la cession en durée et en étendue :

- la cession est souvent et de plus en plus conclue pour la durée de la propriété littéraire et artistique ${ }^{40}$ et cela vaut pour tous les secteurs. Certes, en cas de non-suivi de l'exploitation, la cession est résoluble, mais l'auteur doit engager une action en résolution: serait-il plus simple de limiter le champ temporel de la cession (à 5 ou 10 ans maximum), quitte ensuite, le cas échéant, à la renouveler par tacite reconduction?

- dans le contexte actuel de marchés mondiaux, les droits sont cédés désormais en tous lieux. La question de la pertinence de cette étendue est posée dans un contexte économique où les entreprises sont de moins en moins pérennes (concentration, rachat, disparition pure et simple...), mais aussi du fait de la création de véritables rentes au profit des grands groupes propriétaires d'importants catalogues de droits. Des clauses permettant aux auteurs de reprendre leurs droits en cas de revente des entreprises d'édition pourraient-elles être envisagées?

Le sentiment de dépossession de propriéte éprouvé par les auteurs est encore accru par le fait que, fréquemment, des clauses de non-concurrence par lesquelles s'opère «l'attachement de l'auteur à l'éditeur »-sont introduites dans le contrat ${ }^{41}$, telle la clause d'exclusivité (l'auteur s'engage à ne pas recéder les mêmes droits à quelqu'un d'autre ${ }^{42}$ ), mais surtout la clause de préférence (l'auteur ne signe pas seulement pour une œuvre, mais pour plusieurs ${ }^{43}$ ).

À la différence des auteurs, les artistes interprètes semblent bénéficier d'un statut protecteur ${ }^{44}$. Alors qu'un jugement du tribunal de grande instance de Paris du 4 octobre 1988 considérait que le droit d'autoriser était une modalité du contrat de travail (et donc que le producteur ou employeur pouvait user comme il l'entendait de la force de travail et du produit du travail de son salarié), la Cour de cassation a reconnu en 2001 que le droit du travail ne prévalait pas sur le droit d'autoriser, c'est-à-dire qu'il n'y avait aucune incidence de la qualité de salarié quant à la capacité d'être titulaire de droits voisins ou encore, en d'autres termes, que les droits voisins qui sont reconnus à l'artiste interprète étaient bien des droits individuels et personnels. Cette position offre un éclairage inédit sur l'articulation entre les deux droits. De plus, en 2002 et encore très récemment, en 2006, la Cour a affirmé le pouvoir du droit moral de l'artiste interprète de tempérer la cession exclusive des droits patrimoniaux. Dans le premier cas, c'est sur le terrain du droit à l'intégrité qu'elle donne satisfaction à l'artiste interprète en lui reconnaissant en quelque sorte un droit de divulgation; dans le second, c'est sur celui du droit inaliénable au respect de l'interprétation.

Dans le domaine audiovisuel, la situation des artistes interprètes peut, à certains égards, apparaitre moins favorable. En effet, le Code de la propriété littéraire et artistique (article L. 212-4) transfère, au moyen d'une présomption, la titularité de leurs droits aux producteurs audiovisuels ${ }^{45}$. De plus, la présomption de cession est ici irréfragable, aucune possibilité de clause contraire n'étant prévue.

\section{Répartition des risques et rémunérations}

Cette évolution des droits des travailleurs de la création à travers des situations contractuelles trouve sa concrétisation dans les rémunérations, entendues au sens large, c'est-à-dire comprenant les salaires et les redevances.

69

Partie faible au contrat, le travailleur de la création supporte les conséquences du partage déséquilibré des risques au plan de ses revenus. Cependant les problèmes 
relatifs à la rémunération, lato sensu, sont sans commune mesure pour les travailleurs salariés, au demeurant parfois titulaires de droits voisins, et pour les non-salariés qui ne bénéficient que des fruits de l'exploitation de leurs œuvres. En l'occurrence, les auteurs de l'écrit sont sans doute parmi ceux dont les revenus sont les plus aléatoires, liés au seul succès de l'exploitation. Les artistes plasticiens, dont les revenus principaux procèdent de la vente de leurs œuvres à côté de la cession de leurs droits, voient eux aussi leur situation pécuniaire liée à la valeur marchande de leurs créations. Pour les uns comme pour les autres, la rémunération ne prend aucunement en compte la dimension effective de leur travail. On est face à une coupure structurelle entre le travail du créateur, le produit de ce travail - la création originale - et la rémunération. Cette situation conduit nombre d'artistes, en fait la majorité, à dépendre de l'assistance (RMI) et/ou à rechercher d'autres sources de revenus dans des activités annexes faisant appel à leurs compétences artistiques - des actions de formation par exemple -, voire à exercer des activités non artistiques. Les organismes sociaux comme l'AGESSA et la Maison des artistes (MDA) signalent d'ailleurs une érosion des revenus moyens des $\operatorname{artistes}^{46}$.

\section{Trois questions appellent des régulations juridique}

\section{Comment rémunérer le travail « invisible » des artistes du spectacle?}

70 S'agissant des salariés, la difficulté tient à la prise en compte de la dimension effective du travail dans la rémunération: dans le spectacle vivant comme dans l'audiovisuel ou le cinéma, la rémunération, contrepartie d'une "présence effective ", rétribue-t-elle une quantité/un temps de travail (dont une partie est "invisible») ou plutôt une prestation de services appréciée notamment en fonction de sa qualité et de la notoriété de celui qui l'a produite?

71 L'un des effets inattendus de la crise des intermittents a été de montrer assez clairement que l'assurance chômage couvrait en réalité des situations d'activité dites de "travail invisible », par référence au travail visible qui est celui qui donne lieu à un contrat de travail (ou à un contrat de prestation de services) et à un salaire en contrepartie et qui es mesuré et rémunéré en temps de présence physique. L'existence d'un temps nécessaire à la création a pu être mise en évidence. Ce temps composé d'activités diverses - entretien de la force de travail et des capacités artistiques, assimilation de nouveaux rôles, d'une œuvre ou d'un patrimoine, recherche de contrats, travail de constitution de réseaux, préparation et montage de projets -, certains juristes le qualifient «d'utilité sociale» de l'artiste ${ }^{47}$. Ce travail «invisible » a $\mathrm{pu}$ se développer grâce à une instrumentalisation du droit au chômage et à un glissement du rôle des prestations de chômage. Bien que ce temps d'activité et de nonemploi permette le développement de la création, son financement ne peut continuer d'être pris en charge au travers de la solidarité interprofessionnelle. Par ailleurs, le travail invisible est difficile à qualifier juridiquement entre le travail dissimulé, donc illégal, et le bénévolat, exclusif de professionnalisme.

Au-delà d'une nécessaire redéfinition de la notion de chômage, sans doute faudrait-il en la matière distinguer les situations de succession discontinue d'emplois, fréquentes selon Pierre-Michel Menger $(60 \%$ du travail d'un intermittent est lié à un seul employeur), et les situations de vraie discontinuité d'emploi, non rattachables à un contrat de travail. Lorsque ce temps de travail est rattachable à un contrat, ne pourrait- 
on utilement s'inspirer des solutions trouvées pour des travailleurs, comme les cadres, dont le temps de travail est difficilement comptabilisable (conventions de forfait exprimées en jours)? Lorsqu'il ne l'est pas, ne pourrait-on mutualiser par voie conventionnelle des droits de tirage sociaux dans des assurances spécifiquement créées à cet effet? En tout état de cause, il importerait de favoriser un véritable enchaînement des situations juridiques, une continuité professionnelle par-delà la discontinuité de l'emploi et de permettre d'intégrer ce temps invisible dans le droit social.

\section{Comment répondre à l'attente de sécurité en matière de rémunération de l'exploitation de la création?}

73 Pour les artistes auteurs, la cession des droits patrimoniaux s'accompagne d'une rémunération proportionnelle qui traduit le partage des risques, la rémunération forfaitaire étant censée rester l'exception ${ }^{48}$. La rémunération en droits d'auteur est cruciale en ce qu'elle représente une créance sur l'avenir qui fait que l'on accepte de "travailler longtemps pour pas grand-chose", parce que l'on en espère une contrepartie à la fois symbolique et financière, eu égard à l'impossibilité d'un paiement qui se ferait au temps de travail.

Du fait du décalage structurel entre le travail de l'auteur et sa rémunération en fonction du succès éventuel, on observe en effet un souci rémanent des auteurs de vouloir faire reconnaître et rémunérer le travail aboutissant à la création et d'en sécuriser l'exercice. Ce souci s'exprime sous des formes diverses : dans l'affirmation d'une conception sociale et non artistique des bourses, dans le rappel par le Conseil permanent des écrivains des caractéristiques du travail d'auteur qui s'apparentent au travail intermittent, ou encore dans la revendication de la rémunération des prestations que les auteurs réalisent à l'occasion de foires ou de salons. Ce dernier travail est hautement visible, puisqu'il consiste dans la promotion ou la communication autour de l'œuvre, mais il est « invisible », dans la mesure où il n'est pas rémunéré. Les artistes plasticiens connaissent d'ailleurs le même type de situation en ce qui concerne les expositions. Il s'agit pourtant d'une présentation publique protégée par le droit d'auteur, mais les artistes sont habituellement privés des rémunérations correspondantes et renoncent généralement à leurs droits sur les catalogues.

On assisterait à une progression de la rémunération forfaitaire. En témoignent surtout la pratique courante et, semble t-il, en progression - en particulier dans le domaine littéraire - des à-valoirs et les revendications des écrivains en la matière (leur montant minimal et leur non-report). Ceux-ci restent cependant plutôt faibles, ce qui montre qu'il n'y a pas de rééquilibrage des prises de risque avec le producteur ${ }^{49}$. Dans les contrats d'artistes conclus par les majors, la pratique des avances est également courante. Dans d'autres secteurs, comme celui de la création télévisuelle, la situation est plus favorable: s'ajoutant aux "à-valoirs minimum garantis », il existe une autre rémunération ex ante, qualifiée de "prime de commande ", "prime d'écriture " ou même "somme forfaitaire ", qui rémunère le temps passé à la création de l'œuvre ou encore l'exclusivité accordée au producteur ${ }^{50}$. On est alors dans des modes de rémunération proches du salariat. L'attractivité de la forfaitisation est d'autant plus forte que l'on est face à une tendance de moyen terme de réduction des taux des droits d'auteur. 

et artistique en faveur des artistes interprètes concernant la présomption de cession au producteur s'avèrent réduites, le Code étant imprécis sur le sujet. Il prévoit en effet que le contrat conclu entre un artiste interprète et un producteur pour la réalisation d'une œuvre audiovisuelle fixe une rémunération distincte pour chaque mode d'exploitation, sans donner la définition de ce qu'il faut entendre par "mode d'exploitation». Par ailleurs, la loi indique que, si de telles stipulations n'existent pas dans le contrat individuel, alors la rémunération peut être fixée par voie d'accords collectifs. En d'autres termes, dès lors qu'il y a accord collectif, le contrat vaut cession, même si la rémunération n'est pas fixée. Par glissement donc, l'accord collectif vaut autorisation d'exploiter, ce qui pose un premier problème juridique, les syndicats n'étant pas habilités à donner cette autorisation.

En principe, cette rémunération distincte par mode d'exploitation et la référence à des accords collectifs participent d'une protection en faveur des artistes interprètes. Dans la réalité, la fixation de la rémunération par accord collectif déboucherait sur la pratique de la rémunération globale - minimale ${ }^{51}$ - mélangeant salaire et rémunérations liées aux utilisations secondaires. Cette pratique est rendue possible à la fois par la combinaison des articles L.762-2 du Code du travail ${ }^{52}$ et L. 212-6 du Code de la propriété littéraire et artistique ${ }^{53}$. Elle est en contradiction avec une décision de principe de la Cour de cassation en date du 10 février 1988, qui stipule que "la rémunération de l'artiste interprète au titre de la cession de ses droits sur l'œuvre doit être distincte de la rémunération de sa prestation artistique ». Autrement dit, il convient de distinguer le cachet d'enregistrement, qui rémunère le travail de l'artiste interprète, des redevances liées à l'utilisation dudit enregistrement et qui sont les fruits de l'exploitation de l'interprétation fixée.

urs accords collectifs font ainsi l'objet de critiques comme l'accord spécifique concernant les artistes interprètes engagés pour la réalisation d'une œuvre cinématographique par exemple, l'accord sur le doublage, dit «DAD-R », qui donne également lieu au "saucissonnage » du salaire, pour reprendre le jargon usité par les professionnels, selon les mêmes modalités (imprécision des modes d'exploitation, etc.).

De manière plus générale il apparaît que, dans le domaine audiovisuel, les conventions collectives tendent à assimiler à des salaires des rémunérations qui ressortissent en principe au domaine des redevances: ainsi de la convention collective des artistes interprètes engagés pour des émissions de télévision, de la convention collective des artistes musiciens de la production cinématographique... Cet encadrement de la propriété intellectuelle par le droit du travail est interprété diversement selon les intérêts défendus : manifestation d'une marge de manœuvre réduite des syndicats dans la négociation collective face aux producteurs ou bien, au contraire, facteur d'augmentation des charges sociales pour les employeurs.

Dans le domaine sonore, la situation est encore plus complexe. Dans l'édition phonographique, il n'existe pas de présomption de cession en faveur du producteur - et pas d'obligation subséquente de fixer une rémunération distincte pour chaque mode d'exploitation de l'interprétation de l'œuvre. Suite aux conflits intervenus dans les années 1990 entre le SNAM et la SPEDIDAM d'une part et le SNEP ${ }^{54}$ d'autre part, les artistes interprètes « d'accompagnement » (musiciens, choristes...) sont contraints, par 
le biais des contrats d'enregistrement, de céder tous leurs droits patrimoniaux au producteur. En clair, il s'agit d'une sorte de présomption de cession de facto et non de jure. En revanche, à l'inverse des artistes interprètes "principaux» sous contrats d'artiste, ils ne perçoivent en contrepartie de leur cession globale qu'un salaire forfaitaire et définitif et aucune rémunération proportionnelle au succès de l'exploitation de l'œuvre.

81 Au final, ces évolutions témoignent des difficultés liées à la rémunération du travail de création et, partant, contribuent à renforcer la précarité ainsi que les disparités de revenus entre artistes, du fait du caractère de plus en plus composite et donc variable de ceux-ci. Elles engendrent, dans le même temps, un manque de visibilité globale, pour ne pas dire une opacité, de la situation des différentes catégories de créateurs, que ce soit en termes de partage des revenus entre les différentes modalités de rémunération (salaires/honoraires/droits/...), d'activités principales et secondaires, de secteurs d'activité (culturel/non culturel)...

\section{À l'avenir, quel rôle pour les mécanismes collectifs et pour l'État?}

82 Les décalages constatés entre le droit-légal et réglementaire-et les pratiques conventionnelles - collectives ou individuelles -, les disparités statutaires, l'érosion des revenus moyens des créateurs soulignent la nécessité d'une régulation ayant pour enjeu une conciliation optimale entre l'économique et le social.

L'atteinte de cet objectif suppose de privilégier une approche à la fois organisationnelle (qui structure) et systémique (qui articule des logiques distinctes) plutôt que des approches techniciennes et sectorielles du droit ${ }^{55}$. Elle appelle aussi, dans un contexte de recherche de flexibilité des normes, la prise en compte des différentes catégories d'intérêts dans le cadre de la négociation collective.

Un rapide état des lieux des mécanismes collectifs souligne les faiblesses de ces derniers.

\section{Un système de relations professionnelles aux dynamiques peu structurantes}

Du côté des créateurs salariés, dans les secteurs où la production suppose un travail collectif, le système de relations professionnelles manifeste un émiettement des représentations en raison du maintien de logiques de métiers. Malgré les rassemblements opérés au niveau confédéral, cette dispersion syndicale débouche sur une faible capacité des organisations professionnelles de salariés à jouer leur rôle dans la structuration des professions et dans la négociation avec la partie patronale. Cette dynamique pourrait cependant s'inverser grâce à la réduction récente du nombre d'accords collectifs de branche dans le champ du spectacle

Du côté des investisseurs, l'existence d'une myriade de PME ou TPE auprès de groupes ayant une stature internationale, les premières assumant difficilement leurs responsabilités en matière d'emploi, les seconds ne jouant pas toujours le jeu en la matière, n'est pas non plus favorable à la négociation collective. De plus, le terme usuel d'» entreprise culturelle » recouvre une extraordinaire diversité de statuts juridiques et de réalités économiques, englobant entreprises privées-parfois individuelles -, établissements publics, associations... La représentation des employeurs et donneurs 
d'ordre exprime, elle aussi, les caractéristiques et faiblesses du tissu économique culturel.

87 Ces difficultés conduisent à ce que le cadre des relations collectives de travail peine à apporter les correctifs nécessaires au caractère souvent sporadique de la relation de travail.

\section{Des démarches collectives qui se cherchent chez les non-salariés}

88 L'action collective reste embryonnaire chez les professionnels des secteurs où le travail individuel est dominant. Ainsi de l'écrit, où la très grande diversité des auteurs et de leurs situations au regard du côté professionnel de l'écriture, littéraire ou non, la multiplicité des écritures et des parcours, les conditions de l'écriture ainsi que la gestion restée individuelle des droits valorisent l'auteur dans sa singularité, avec ses particularités, au détriment de solidarités potentielles. Dans ces conditions, il existe peu d'organisations de représentation collective en dehors de la "légitimité fédérative » du Conseil permanent des écrivains, de la SGDL, du SNAC, et d'un nouvel acteur syndical, le SCEI qui milite pour une convention collective et préconise un droit d'auteur fixe et garanti, en plus de la rémunération proportionnelle.

89 Face à la situation d'isolement et de sous-information dans leur situation professionnelle, les créateurs indépendants peuvent trouver intérêt à la mise en place de structures de représentation collective, pour l'heure faite de collectifs et de réseaux, comme c'est le cas pour les scénaristes ou plasticiens. Ces derniers viennent de se regrouper dans l'» union des associations et des organisations professionnelles des arts visuels ", tandis que la Maison des artistes a engagé des démarches de recensement incitant à la déclaration et permettant de passer «du métier à la profession ». La création récente - dans le contexte du numérique - de la Société des auteurs des arts visuels et de l'image fixe (SAIF) participe de la même dynamique. Rien n'empêcherait ces travailleurs indépendants de conclure des accords collectifs de droit civil car le droit à la négociation collective est un droit constitutionnel qui n'est pas réservé aux salariés. Le législateur pourrait d'ailleurs inciter à cette dynamique comme cela a été fait au Canada avec la loi sur le statut des artistes qui met en place un système de relations collectives pour les artistes indépendants, et même un tribunal chargé d'arbitrer les différends surgis dans le cadre de la négociation collective.

\section{L'action collective via la gestion collective des droits d'auteur}

90 La gestion collective des droits d'auteur et droits voisins constitue un autre mécanisme collectif important. Elle est reconnue comme un instrument utile de protection individuelle et d'équilibre des rapports de force collectifs, mais la gestion individuelle des droits reste courante voire prédominante dans certains secteurs, comme l'édition de livres où prévalent quelques codes d'usage professionnels et une gestion collective embryonnaire. Cependant, même dans ce secteur, la gestion collective est appelée à se développer pour assurer la gestion des nouveaux droits à rémunération (SOFIA). L'intérêt pour cette forme de gestion a plusieurs explications : elle s'impose en raison des limites de la gestion individuelle des droits du fait du mécanisme de la demande préalable d'autorisation (à ce titre, elle apporte une simplification au profit des utilisateurs des droits) ; elle permet ensuite de faire bénéficier les artistes de moyens communs, moyens de perception, mais aussi juridiques permettant de mieux garantir 
leurs revenus, ce qui augure de droits mieux défendus ; elle favorise donc un rééquilibrage dans la relation contractuelle. Enfin, les SPRD jouent un rôle non négligeable tant dans l'aide sociale que dans l'aide à la création, en particulier au travers des actions d'intérêt général. La gestion collective est déjà obligatoire pour un certain nombre de droits depuis la loi de 1985. Faut-il aller plus loin? Son avenir relevant de plus en plus de directives communautaires, comment se concilieront les finalités économiques et les finalités sociales de ce mécanisme de représentation d'intérêts collectifs ?

\section{L'État, arbitre des régulations du travail artistique?}

91 L'incitation à l'action collective est un enjeu important pour la puissance publique même si l'État ne peut pas toujours facilement jouer le rôle de "tiers garant ${ }^{56}$ ". Il se trouve en effet dans l'obligation d'intervenir dans un domaine qui relève de la responsabilité des partenaires sociaux, au travers du paritarisme. Par ailleurs, dans le domaine de la culture, il assume un double rôle de médiateur et de financeur.

L'intervention attendue de la part de l'État pourrait consister à accompagner l'émergence de nouveaux acteurs, mettre en place de nouvelles scènes de négociation..., en vue de susciter une nouvelle dynamique d'action collective.

\section{Il pourrait :}

- continuer à inciter les partenaires sociaux à actualiser les conventions collectives, non seulement en termes de champ couvert, mais aussi en termes de contenus - la mutualisation des droits sociaux, les conditions effectives de l'exercice de l'activité, les règles non dérogeables à la juste rémunération du travail de création et de ses fruits ;

- pousser à la création ou la refondation de structures de représentation collective conférant aux professions, par leur capacité à fédérer, une visibilité extérieure accrue. Celles-ci pourraient avoir vocation à fournir informations et conseils, à offrir des espaces d'échanges et de débats, à optimiser l'identification des besoins et l'accès à la formation. Se poserait alors la question de l'articulation, voire de la compatibilité, entre ces structures et celles ayant vocation à gérer collectivement les droits des auteurs (ou des auxiliaires de la création) ou la gestion mutualisée de la sécurité sociale des professions;

- amener les acteurs d'une même filière à définir ensemble des accords collectifs de droit civil qui, comme nous l'avons vu plus haut, peuvent permettre aux « indépendants » de se doter des droits sociaux dont ils ne bénéficient pas aujourd'hui;

- adopter en la matière une posture régulatrice développée par ailleurs sur d'autres aspects et articulations cruciales des filières ;

- favoriser une réflexion juridique de fond sur les besoins d'un encadrement juridique renouvelé de la création et du travail de création.

Telles sont quelques-unes des diverses voies qui peuvent être explorées. 


\section{NOTES}

1. Pour une revue de la littérature sur la "flexicurité », voir METIS, Correspondances européennes du travail, $\mathrm{n}^{\circ}$ 10, août 2007. http://metiseurope.eu/ARTN08 FLEXICURIT_flexicurite.html

2. Pierre-Michel MENGER, Portrait de l'artiste en travailleur, Paris, La République des idées/Le Seuil, 2002.

3. Ibid., p. 8.

4. Xavier GREFFE, Arts et artistes au miroir de l'économie, Paris, Unesco/Economica, 2002.

5. Pascal NICOLAS-LE STRAT, Une sociologie du travail artistique, Paris, L'Harmattan, coll. « Logiques sociales », 1998.

6. Économiquement, les droits d'auteur ont deux fonctions : inciter à la création et à la production d'œuvres tout en préservant leur usage (droits exclusifs et temporaires) ; faciliter les échanges (droits cessibles).

7. Voir Anne LATOURNERIE, « Petite histoire des batailles du droit d'auteur », Multitudes, mai 2001. L'auteur rappelle dans cet article l'objectif du projet de loi relatif au droit d'auteur déposé par Jean Zay, ministre de l'Éducation nationale et des Beaux-arts en 1936, tendant à instituer un statut des «travailleurs intellectuels».

8. Article L.762-1 du Code du travail : "Sont considérés comme des artistes du spectacle, notamment l'artiste lyrique, l'artiste de variétés, le musicien, le chansonnier, l'artiste de complément, le chef d'orchestre, l'arrangeur-orchestrateur et, pour l'exécution matérielle de sa conception artistique, le metteur en scène. "

9. Si le statut de salarié s'applique, à l'instar de la France, en Belgique ou en Espagne, dans la plupart des autres pays, l'emploi dans le champ du travail artistique, du fait de ses particularités, ne se réfère pas nécessairement pour autant au statut de travailleur indépendant mais plutôt à des formules mixtes : para-subordination (Italie, Allemagne, Autriche), statut d'entrepreneur avec une licence spécifique (Suède). Voir Le régime d'emploi et de protection sociale des travailleurs du spectacle et de l'audiovisuel dans les pays membres de l'Union européenne, DGEAC, CES, 2003.

10. Code du travail, article L.762.1

11. Code de la sécurité sociale, article L.311-3-15 qui prévoit que « les artistes du spectacle sont l'une des catégories de personnes affiliées au régime général des assurances sociales dès lors que leur sont applicables les dispositions des articles L.762-1 et suivants du Code du travail ».

12. Il s'agit « d'artistes auteurs d'œuvres littéraires et dramatiques, musicales et chorégraphiques, audiovisuelles et cinématographiques, graphiques et plastiques ainsi que photographiques ». L'article R.382-2 du Code de la sécurité sociale donne une liste des activités rattachées par branche professionnelle donnant lieu à affiliation au régime général de la sécurité sociale.

13. La loi n 75-1348 modifiée du 31 décembre 1975 sera d'ailleurs la dernière loi d'extension des assurances sociales à des professions non typées comme salariées.

14. Marie-Laure MORIN, Prestation de travail et activité de service, Paris, La Documentation française, 1999. L'auteur distingue quatre types de risques : risque physique sur le travail, risques économiques qui se décomposent en risque d'entreprendre (risque sur le patrimoine) et risques de marché (incertitude sur la valorisation par le marché des produits et services offerts, risque clientèle), risque d'emploi. Les risques économiques et d'emploi sont supportés par l'employeur dans le cadre du salariat, mais dans des situations de "travail limité » et/ou de travail dans lequel le travailleur dispose d'une forte autonomie, un rapprochement s'opère entre travailleur salarié et travailleur indépendant quant au risque d'emploi et aux risques économiques.

15. Nathalie SONNAC, Économie des industries audiovisuelles, Paris, La Découverte, 2006. 
16. Thomas PARIS, Organisation, processus et structures de la création, Paris, Ministère de la culture et de la communication, DEPS, coll. «Culture prospective », 2007-5.

17. Françoise BENHAMOU, L'économie du star-system, Paris, Odile Jacob, 2002.

18. Françoise BENHAMOU, Dominique SAGOT-DUVAUROUX, La place et le rôle de la propriété littéraire et artistique dans le fonctionnement économique des filières d'industrie culturelle, Paris, Ministère de la culture et de la communication, DEPS, avril 2007 (rapport d'étude). Une synthèse de ce rapport fera l'objet d'un numéro de "Culture études ", diffusé en décembre 2007 sur www.culture.gouv.fr/deps (rubrique : publications).

19. Philippe CHANTEPIE, Alain LE DIBERDER, Révolution numérique et industries culturelles, Paris, La Découverte, coll. « Repères », 2005. Voir aussi Philippe CHANTEPIE, « L'accès : convergence des régulations des plates-formes numériques ?", dans Création et diversité au miroir des industries culturelles, actes des Journées d'économie de la culture coordonnées par Xavier GREFFE (12-13 janvier 2006), Paris, Ministère de la culture et de la communication, DEPS, coll. «Questions de culture ", 2006, p. 373-411.

20. Entendu notamment (art. L 762-1 du Code du travail) comme "l'artiste lyrique, l'artiste dramatique, l'artiste chorégraphique, l'artiste de variétés, le musicien, le chansonnier, l'artiste de complément, le chef d'orchestre, l'arrangeur-orchestrateur et, pour l'exécution matérielle de sa conception artistique, le metteur en scène ».

21. Art. L 212-1 du Code de la propriété littéraire et artistique : « À l'exclusion de l'artiste de complément, considéré comme tel par les usages professionnels, l'artiste interprète ou exécutant est la personne qui représente, chante, récite, déclame, joue ou exécute de toute autre manière une œuvre littéraire ou artistique, un numéro de variétés, de cirque ou de marionnettes. »

22. Le Code du travail fait référence à « la personne physique ou morale qui s'assure le concours d'un artiste du spectacle ».

23. Pierre-Michel MENGER, Les intermittents du spectacle. Sociologie d'une exception, Paris, Éditions EHESS, 2005.

24. Emmanuel PIERRAT, " L'auteur, une notion à géométrie très variable ", Propriétés intellectuelles, juillet $2002, \mathrm{n}^{\circ} 4$.

25. B. DUBOURDIEU, Le statut social de l'artiste, thèse sous la direction de Bernard Teyssié, Paris, Université de Panthéon-Assas, 1988. " La justification de cette inégalité n'apparaît pas dans les textes de propriété littéraire et artistique. Elle semble aller de soi et, pour cette raison, présente de nombreux aspects ambigus. »

26. Article L 762-2 du Code du travail : « N’est pas considérée comme salaire la rémunération due à l'artiste à l'occasion de la vente ou de l'exploitation de l'enregistrement de son interprétation, exécution ou présentation par l'employeur ou tout autre utilisateur dès que la présence physique de l'artiste n'est plus requise pour exploiter ledit enregistrement et que cette rémunération n'est en rien fonction $\mathrm{du}$ salaire reçu pour la production de son interprétation, exécution ou présentation, mais au contraire fonction du produit de la vente ou de l'exploitation dudit enregistrement. "

27. Dans la majorité des pays européens, le travail artistique est considéré comme une activité indépendante, même s'agissant des prestations relevant des secteurs du spectacle, vivant ou enregistré. Un recours avait été introduit contre la France par la Commission européenne considérant que la législation française sur les licences des agences de placement des artistes d'une part et sur la présomption de salariat des artistes du spectacle (art. L.762-1 du Code du travail) constituait une entrave à la libre prestation de service au sein de l'Union européenne. La Cour de justice des Communautés européennes, dans un arrêt du 15 juin 2006, a considéré que la présomption de salariat n'a pas à être remise en cause, sauf si elle est imposée aux artistes qui sont reconnus comme prestataires de services établis dans leur État membre d'origine où ils fournissent habituellement des services analogues et qui viennent exercer en France à titre temporaire et indépendant. 
28. Le louage d'ouvrage est défini par l'article $1710 \mathrm{du}$ Code civil comme « le contrat par lequel l'une des parties s'engage à faire quelque chose pour l'autre moyennant un prix convenu entre elles ». Voir à ce sujet Marie-Laure MORIN, « Louage d'ouvrage et contrat d'entreprise », dans Le travail en perspectives, Paris, LGDJ, coll. " Droit et société », 1998. Historiquement, en 1854, Augustin Charles Renouard proposa de remplacer ce terme par celui de « contrat de prestation de travail », contrat dans lequel la subordination n'avait rien à faire.

29. Juliette MEL, « Le photographe de presse aux confins du droit d'auteur et du droit du travail ", La semaine juridique. Édition sociale $\mathrm{n}^{\circ} 22$, novembre 2005.

30. «Une substitution à grande échelle a été opérée, qui s'ajustait à des choix organisationnels et économiques de réduction des coûts fixes de main-d'œuvre, d'augmentation de la flexibilité interne et de production par relations de coopération et de sous-traitance entre entreprises : ce sont les caractéristiques de la désintégration verticale de l'activité ", dans P.-M. MENGER, Les intermittents du spectacle. Sociologie d'une exception..., op. cit..

31. Cour de cassation, chambre sociale, arrêts du 26 novembre 2003, $n^{\text {os }} 2663,2664,2665$ et 2666.

32. Art L122-1 du Code du travail : le contrat de travail à durée déterminée, quel que soit son motif, ne peut avoir ni pour objet ni pour effet de pourvoir durablement un emploi lié à l'activité normale et permanente de l'entreprise. Sous réserve des dispositions de l'article L. 122-2, il ne peut être conclu que pour l'exécution d'une tâche précise et temporaire, et seulement dans les cas énumérés à l'article L. 122-1-1.

33. De cette « libération d'activité » a résulté une prolifération de petites entreprises, notamment dans le spectacle vivant, des structures associatives certes culturellement peu préparées à une fonction d'employeur, dans l'audiovisuel des petites, voire très petites entreprises de prestations de services, qui se sont développées très rapidement. Cette multiplication de structures entrepreneuriales a aussi vraisemblablement produit un effet positif en amplifiant le nombre de biens et services culturels proposés aux usagers, pratiquants et consommateurs culturels.

34. P.-M. MENGER, Les intermittents du spectacle. Sociologie d'une exception..., op. cit.

35. Accord Michel du 12 octobre 1988 : «L'employeur d'un salarié sous CDD d'usage ne peut imposer à celui-ci, pour ce qui est de la durée du contrat, une incertitude supérieure à celle qui pèse sur l'entreprise pour l'objet du contrat. »

36. Christophe GEIGER, Droit d'auteur et droit du public à l'information, Paris, Litec, 2004.

37. Ainsi, début 2000, un groupe d'auteurs animé par Ayerdhal, écrivain de science-fiction, avait lancé un manifeste sur " le droit du serf » et revendiquait l'établissement d'un contrat d'exploitation, à caractère plus limité et mieux spécifié, en lieu et place du contrat, en l'occurrence, d'édition : "Il nous paraît urgent d'informer les citoyens et les élus de la très féodale réalité découlant de ces fameux droits. En commençant par préciser que les droits sur l'ouvrage n'appartiennent pas à l'écrivain mais à l'éditeur. Car la véritable spoliation s'effectue lors de l'établissement du contrat quand, pour être publié, l'auteur est contraint de céder ses droits patrimoniaux à l'éditeur, tous ses droits, depuis celui de publier l'ouvrage sous sa forme livresque jusqu'à ceux de traduction, de reproduction et d'adaptation par tout procédé. »

38. À l'exception des droits d'adaptation visuelle qui font toujours l'objet d'un contrat distinct.

39. Le Code de la propriété intellectuelle fixe les clés de répartition en ce qui concerne la rémunération équitable et la rémunération pour copie privée sonore, audiovisuelle, de l'écrit et de l'image fixe.

40. Soixante-dix ans après le décès de l'auteur.

41. Ces clauses sont donc invalides au regard de ce droit de la concurrence et, par ailleurs, discutables du point de vue de leur compatibilité avec le droit moral de l'auteur.

42. La clause catalogue pour les artistes interprètes est de même nature : interdiction de réenregistrer pendant un certain temps. 
43. Jean-Yves KERBOURC'H, Jean VINCENT, « Jusqu'où les contrats de production et d'édition conclus par des artistes de la musique peuvent- ils restreindre leur liberté de travailler ? ", Droit social, $\mathrm{n}^{\circ} 7-8$, août 2007.

44. Certains aspects de leur statut «pourraient servir de modèle au statut de l'auteur ", écrit Bernard Edelman, qui estime qu'ils « pourraient constituer désormais une avant-garde [dans le combat contre le copyright] ». Voir Bernard EDELMAN, Le sacre de l'auteur, Paris, Le Seuil, 2004.

45. « Dans le cadre strict de l'audiovisuel, le législateur restaure la règle civiliste de l'accession en ce qui concerne les fruits du travail salarié. En l'occurrence [...] le contrat de travail que signe l'artiste interprète altère sa capacité à exercer ses droits voisins. Ce sera donc le producteur audiovisuel qui sera à même de les exercer. " Stéphane PESSINA-DASSONVILLE, L'artiste interprète salarié. Entre création intellectuelle et protection sociale, thèse de droit privé, Montpellier, Université de Montpellier, décembre 2004.

46. Les artistes plasticiens n'ont pas réussi à ce jour à faire reconnaître ces revenus comme revenus artistiques, ce qui a bien sûr des incidences non négligeables du point de vue de leur protection sociale. Voir Écrivains, photographes, compositeurs... les artistes auteurs affiliés à l'AGESSA en 2005, Paris, Ministère de la culture et de la communication, DEPS, coll. "Culture chiffres ", 2007-5 ; et Peintres, graphistes, sculpteurs... les artistes auteurs affiliés à la Maison des artistes en 2005, Paris, Ministère de la culture et de la communication, DEPS, coll. « Culture chiffres », 2007-6. Voir www.culture.gouv.fr/deps (rubrique : publications statistiques).

47. Isabelle DAUGAREILH, Philippe MARTIN, « Les intermittents du spectacle : une figure du salariat entre droit commun et droit spécial ", Revue française des affaires sociales, nº 3-4, 2000.

48. En cas de difficultés de calcul ou de la nature et des conditions de l'exploitation, en ce qui concerne les œuvres collectives et certains types d'ouvrages ou illustrations de ceux-ci.

49. Néanmoins dans l'édition, la pratique est de les fixer à un niveau qui correspond aux droits de la moitié du premier tirage ou, en poche, au premier tirage tout entier.

50. Françoise BENHAMOU, Stéphanie PELTIER, Économies des droits d'auteur. 2. La filière audiovisuelle, Paris, Ministère de la culture et de la communication, DEPS, coll. " Culture études ", 2007-5. À paraître en décembre 2007 sur www.culture.gouv.fr/deps (rubrique : publications).

51. Le « minimum syndical».

52. Les dispositions de l'article L. 762-2 du Code du travail ne s'appliquent qu'à la fraction de la rémunération versée en application du contrat excédant les bases fixées par la convention collective ou l'accord spécifique.

53. N'est pas considérée comme salaire la rémunération due à l'artiste à l'occasion de la vente ou de l'exploitation de l'enregistrement de son interprétation, exécution ou présentation par l'employeur ou tout autre utilisateur dès lors que la présence physique de l'artiste n'est plus requise pour exploiter ledit enregistrement et que cette rémunération n'est en rien fonction du salaire reçu pour la production de son interprétation, exécution ou présentation, mais au contraire fonction du produit de la vente ou de l'exploitation dudit enregistrement.

54. Dénonciation en 1993 par le SNEP du protocole d'accord signé le $1^{\mathrm{er}}$ mars 1969 qui fixait les conditions de travail et de rémunération des artistes musiciens et des artistes musiciens chanteurs dans l'industrie phonographique.

55. Sur ces approches, voir par exemple Jacques BARTHÉLÉMY et Gilbert CETTE, « Réformer et simplifier le droit du travail via un rôle accru du droit conventionnel », et sur l'hypothèse d'un droit de la régulation, Fabrice SIIRIAINEN, " "Droit d'auteur" contra "droit de la concurrence” : versus "droit de la régulation" ", Revue internationale de droit économique, 2001.

56. Alain SUPIOT, « La contractualisation de la société », Courrier de l'environnement, $\mathrm{n}^{\circ} 43$, mai 2001. 